\title{
ОПТИМАЛЬНОЕ УПРАВЛЕНИЕ ПРОЦЕССОМ ПРОИЗВОДСТВА ФОРМАЛЬДЕГИДА ИЗ МЕТАНОЛА
}

\author{
Часть I
}

Излагаются результаты исследования законов управления квазистационарным химическим процессом. Работа состоит из двух частей. В первой части дается постановка задачи управления и методика математического моделирования процесса с оценкой точности модели. Вторая часть посвящена численному расчету оптимальных режимов конкретного процесса и будет опубликована отдельно.

При пронзводстве формальдегида, как и при многих других квазистационарных каталитических процессах, возникает задача определения оптимальных законов изменения режимов во времени. Активность катализатора непрерывно изменяется, и в определенный момент эксплуатация данной массы катализатора становится экономически нецелесообразной. Этот момент определяет срок службы катализатора. Качество управления процессом оценивается рядом экономических и технологических показателей. Каждому состоянию катализатора соответствует вполне определенная оптимальная комбинация состава исходной смеси и нагрузки на катализатор.

Расчет оптимальных в каком-нибудь смысле режимов контактного процесса представляет собой типичную задачу теории оптимального управления. Успешность ее решения в значительной степени предопределяется адекватностью имеющейся математической модели исследуемому процессу, в связи с чем основное внимание в первой части работы будет уделено конструнрованию математической модели, пригодной для целей управления.

\section{1. Постановка задачи}

В качестве объекта управления рассмотрим контактный аппарат с неподвижным слоем катализатора, через которой под избыточным давлением проходит смесь паров воды и метанола с воздухом. Управляющими воздействиями являются концентрации (или парциальные давления) метанола и кислорода в парах и нагрузка на катализатор. Обычно вместо концентраций рассматриваются весовые соотношения воздуха, воды и метанола в парах. Совокупность управляющих воздействий обозначим вектором управлений $x=\left(x_{1}, \ldots, x_{p}\right)$.

Результат контактного процесса характеризуется вектором $\eta=\left(\eta_{1}, \ldots, \eta_{q}\right)$, координатами $\eta_{j}$ которого являются компоненты контактного газа, температура в зоне контактирования, выход формальдегида, производительность и др. Благодаря старению катализатора вектор $\eta$ явно зависит и от времени $t$. Будем рассматривать вектор-функцию $\eta\left(x_{1}(t), \ldots, x_{p}(t)\right)$ на интервале времени $[0, T]$, равном циклу службы катализатора.

Нас будут интересовать лишь следующие координаты, необходимые для решения задачи управления: 
$\eta_{g}=\eta_{g}(x, t)$ - производительность по $\beta_{f} \%$-ному формалину, тонн в единицу времени;

$\boldsymbol{\eta}_{k}=\eta_{k}(x, t) \quad$ - затраты на производство $\eta_{g}$ тонн формалина, руб.;

$\eta_{c}=\eta_{c}(x, t) \quad$ - себестоимость тонны формалина, руб.;

$\eta_{\Phi}=\eta_{\Phi}(x, t)$ - выход формальдегида на пропущенный метанол;

$\eta_{\Theta_{k}}=\eta_{\Theta_{k}}(x, t)$ - измеряемая температура в зоне контактирования, ${ }^{\circ} \mathrm{C}$;

$\eta_{\gamma}=\eta_{\gamma}(x, t) \quad$ - соотношение содержания метанола к формальдегиду в контактном газе.

Очевидно, что $\eta_{k}(x, t)=\eta_{g}(x, t) \eta_{c}(x, t)$.

Величина $\eta_{c}$ определяется ценой метанола $h_{m}$, требуемым содержанием формальдегида в формалине $\beta_{f}$, выходом формальдегида $\eta_{\Phi}$ и постоянной составляющей себестоимости $c^{0}$ :

$$
\eta_{c}(x, t)=\frac{\beta_{f} h_{m}}{\eta_{\Phi}(x, t)}+c^{0}
$$

Компоненты вектора $x$ :

$x_{1}$ - отношение содержаний кислорода и метанола в парах; $x_{2}$ - отношение содержаний воды и метанола; $x_{3}$ - нагрузка на катализатор, тонн в единицу времени.

Наибольший практический интерес для производства представляет задача нахождения управлений в функции времени, обеспечивающих заданный объем производства формалина заданного качества за цикл при минимально возможных затратах. Следовательно, математическая формулировка задачи имеет следующий вид: найти вектор-функцию времени $x^{*}(t), 0 \leqslant t \leqslant T^{*}$, удовлетворяющую условиям (1.1)-(1.7).

$$
\int_{0}^{T^{*}} \eta_{k}\left(x^{*}(t), t\right) d t=\min _{x \in X} \int_{0}^{T^{*}} \eta_{k}(x(t), t) d t
$$

- условие минимума затрат на интервале $[0, T]$;

$$
\int_{0}^{T^{*}} \eta_{g}\left(x^{*}(t), t\right) d t=G^{T^{*}}
$$

- условие заданного объема выпуска продукции на том же интервале. Допустимая область управления $X$ задается следующими условиями:

$$
\gamma^{\prime} \leqslant \eta_{\gamma}(x(t), t) \leqslant \gamma^{\prime \prime}
$$

- условие заданного качества продукции;

$$
\eta_{\Theta_{k}}(x(t), t) \leqslant \Theta_{k}^{0}
$$

- условие термической устойчивости катализатора;

$$
x_{1}^{\prime} \leqslant x_{1}(t) \leqslant x_{1}^{\prime \prime}
$$

- условие взрывобезопасности;

9 ENSV TA Toimetised F-3 65. 


$$
x_{2}^{\prime} \leqslant x_{2}(t) \leqslant x_{2}^{\prime \prime}
$$

- условие технологической осуществимости процесса;

$$
x_{3}^{\prime} \leqslant x_{3}(t) \leqslant x_{3}^{\prime \prime}
$$

- условие механической устойчивости катализатора.

Неравенства (1.4) - (1.7) представляют условия физической осуществимости управлений.

Число $T^{*}$ не фиксировано заранее, а определяется из условия минимума средней себестоимости за цикл (1.8), включая затраты $K^{0}$ за время $W$ простоя агрегата и на замену катализатора, которые принимаются постоянными:

$$
\frac{\int_{0}^{T^{*}} \eta_{k}\left(x^{*}(t), t\right) d t+K^{0}}{\int_{0}^{T^{*}} \eta_{g}\left(x^{*}(t), t\right) d t}=\min _{T} \frac{\int_{0}^{T} \eta_{k}\left(x^{*}(t), t\right) d t+K^{0}}{\int_{0}^{T} \eta_{g}\left(x^{*}(t), t\right) d t} .
$$

$G^{T}$ определяется, исходя из производственного плана $G^{P}$ на отчетный период $P$ (квартал, год) при фиксированных $W$ и $T$.

Очевидно

$$
G^{T}=\frac{G^{P} T}{P-W_{\Sigma}}
$$

Суммарное время простоя $W_{\Sigma}$ на $P$ зависит от параметра $\omega$ (сдвига начала цикла) $; \omega-$ случайная величина $0 \leqslant \omega \leqslant T$. Можно показать, что математическое ожидание $W_{\Sigma}$ при равномерном распределении вероятностей $\omega$ выражается как

$$
M\left\{W_{\Sigma}\right\}=W \frac{P}{W+T} .
$$

Текущее состояние процесса в момент $t$ характеризуется выработкой формалина $G(t)$ и суммарными расходами $K(t)$ с начала цикла до момента $t$ :

$$
\begin{aligned}
& G(t)=\int_{0}^{t} \eta_{g}(x(t), t) d t \\
& K(t)=\int_{0}^{t} \eta_{k}(x(t), t) d t .
\end{aligned}
$$

Задача отыскания минимума величины $K^{\prime}(T)$ при условиях (1.2) (1.7) сводится к максимизации некоторой функции $H(x(t), \psi(t), t)$ согласно принципа максимума для неавтономных систем [1], т. е.

$$
H\left(x^{*}(t), \psi(t), t\right)=\max _{x \in X} H(x(t), \psi(t), t),
$$


причем

$$
H=\psi_{k} \eta_{k}(x(t), t)+\psi_{g} \eta_{g}(x(t), t) .
$$

Здесь $\psi_{g}$ - решение уравнения

$$
\frac{d \psi_{g}}{d t}=-\frac{\partial H(x(t), \psi(t), t)}{\partial G(t)} .
$$

В простейшем случае, когда координаты $\eta_{j}$ не зависят от $G$, будет $\psi_{g}=$ const. $\psi_{k}$ является произвольной отрицательной константой, прннимаем $\psi_{k}=-1$.

$G(T)$ будет функцией $\psi_{g}$, если при каждом $t$ выполняется (1.11), поэтому $\psi_{g}$ находится согласно (1.2) решением уравнения

$$
G\left(\psi_{g}, T\right)-G^{T}=0
$$

Таким образом, (1.12) принимает вид

$$
H=\psi_{g} \eta_{g}(x(t), t)-\eta_{k}(x(t), t) .
$$

Отметим, что выражение (1.14) формально тождественно выражению «чистого дохода» в единицу времени, причем «цена» продукта равна $\Downarrow_{g}$ и должна рассчитываться исходя из заданного производственного плана, согласно (1.13). Таким образом, исходная задача (условия (1.1) - (1.7)) сводится к задаче максимизации «чистого дохода» (1.14) в каждом фиксированном малом интервале времени.

Уравнения

$\eta_{g}=\eta_{g}(x(t), t), \quad \eta_{\Phi}=\eta_{\Phi}(x(t), t), \quad \eta_{\gamma}=\eta_{\gamma}(x(t), t), \quad \eta_{\theta_{k}}=\eta_{\Theta_{k}}(x(t), t)$,

а также условия (1.2) - (1.7) представляют математическую модель $M$ процесса и могут быть получены либо чисто статистическим (эмпирическая модель $M_{e}$ ), либо теоретическим методом в комбинации со статистическим анализом (теоретическая модель $\left.M_{t}\right)$.

Применение $M$ в целях управления предопределяет некоторые особенности ее составления. Требуемая точность определения отдельных координат различна, находясь в зависимости от их физического смысла. Так, условия физической осуществимости $(1.4),(1.5),(1.6),(1.7)$ должны выполняться с заданной вероятностью для каждого индивидуального наблюдения (критичные условия), в то время как экономические условия (1.2) и (1.3) должны выполняться с заданной вероятностью для средних значений совокупностей наблюдений (некритичные условия).

Немаловажное значение имеет также определение области возможных значений $\tilde{X}$, в пределах которой $M$ имеет заданную адекватность управляемому объекту.

\section{2. Модель $M_{e}$}

Эмпирическая модель для управления процессом представляется системой линейных уравнений регрессии

$$
y_{i}=b_{0 j}+\sum_{i=1}^{p} b_{i j} x_{i} \quad(j=1,2, \ldots, q),
$$


которая является оценкой гипотетической системы уравнений

$$
\eta_{i}=\beta_{0 j}+\sum_{i=1}^{p} \beta_{i j} x_{i} \quad(i=1,2, \ldots, q) .
$$

Применению $M_{e}$ для целей управления должен предшествовать количественный анализ точности модели. Вопросам точности моделей управления в литературе не уделялось должного внимания, хотя применение $M_{e}$ без обоснованных доверительных интервалов лишено всякого смысла.

Следует различать два типа доверительных интервалов значений функций:

1) фидуциальный интервал, попадание в который средних значений $\eta_{i}$ при фиксированных $x_{i}$ гарантируется с заданной вероятностью $1-\alpha$; двухсторонний фидуциальный интервал ограничен сверху функцией $y_{j}+t_{\frac{\alpha}{2}, N-p-1} \Delta_{j}^{f}$ и снизу функцией $y_{j}-t_{\frac{\alpha}{2}, N-p-1} \Delta_{j}^{f}(f$-границами $) ;$

2) толерантный интервал, попадание в который индивидуальных значений $\eta_{j}$ при фиксированных $x_{i}$ гарантируется с заданной вероятностью $1-\alpha$. Двухсторонний толерантный интервал ограничен сверху функцией $y_{j}+t_{\frac{\alpha}{2}, N-p-1} \Delta_{j}^{\tau}$ и снизу функцией $y_{j}-t_{\frac{\alpha}{2}, N-p-1} \Delta_{j}^{\tau}$ ( $\tau$-границами). $t_{\frac{\alpha}{2}, N-p-1}$ есть $100 \frac{\alpha}{2}$-процентная точка $t$-распределения с $N-p-1$ степенями свободы.

Для построения доверительных интервалов многомерного регрессионного уравнения введем обозначение

$$
A=\left\|a_{i h}\right\|, \text { причем } a_{i h}=\sum_{i=1}^{N} \sum_{h=1}^{N}\left(x_{i}-\bar{x}_{i}\right)\left(x_{h}-\bar{x}_{h}\right) .
$$

Как известно $\left[{ }^{2}\right]$, при многомерной регрессии толерантные интервалы для $\eta_{j}$ определены функцией

$$
\Delta_{j}^{\tau}=s_{j} \sqrt{1+\frac{1}{N}+\sum_{i=1}^{p} \sum_{h=1}^{p}\left(x_{i}-\bar{x}_{i}\right)\left(x_{h}-\bar{x}_{h}\right) a^{i h}}
$$

и фидуциальные интервалы для $\eta_{j}-$ функцией

$$
\Delta_{j}^{f}=s_{j} \sqrt{\frac{1}{N}+\sum_{i=1}^{p} \sum_{h=1}^{p}\left(x_{i}-\bar{x}_{i}\right)\left(x_{h}-\bar{x}_{h}\right) a^{i h}},
$$

где $s_{j}$ - остаточное стандартное отклонение; $N$ - число наблюдений; $a^{\text {ih }}$ - элемент матрицы $A^{-1} ; \bar{x}_{i}, \bar{x}_{h}-$ средние значения.

В случае построения односторонней границы берем $100 \alpha$-процентную точку $t$-распределения с $N-p-1$ степенями свободы.

Предлагается использовать $\tau$ - и $f$-границы следующим образом. Если условиями задачи управления какая-либо функция $\eta_{j}$ ограничена, то в модели в качестве ее оценки примем $\tau$-границу при критичных условиях и $f$-границу - при некритичных условиях, с учетом знака неравенства ограничения. Этим гарантируется выполнение условий исходной задачи с заданной вероятностью $1-\alpha$. 
Ограничения поставленной задачи (1.3)-(1.7) определяют допустимую с точки зрения технологических и экономических соображений область $X$. Кроме того, свойства исходного статистического материала опре-деляют некоторую область $\tilde{X}_{j}$, внутри которой уравнения регрессии имеют заданную точность. Максимально допустимое стандартное отклонение среднего значения $\left(\Delta_{j}^{f}\right)_{\max }=\frac{\tilde{s}_{j}}{\sqrt{N}}$ определяет границу фидуциальной допустимой области $\tilde{X}_{i}$ :

$$
\left(\Delta_{j}^{f}\right)_{\max }=\frac{\tilde{s}_{j}}{\sqrt{N}}=\frac{s_{j}}{\sqrt{N}} \sqrt{1+N \sum_{i=1}^{p} \sum_{h=1}^{p}\left(x_{i}-\bar{x}_{i}\right)\left(x_{h}-\bar{x}_{h}\right) a^{i h} .}
$$

Рассмотрим $\eta_{u}, u=1,2, \ldots, q-r$, где $r$ - число координат $\eta$, определяющих область $X$. Обозначим

$$
\frac{\tilde{s}_{u}^{2}}{s_{u}^{2}}=\beta_{u} .
$$

Тогда $\beta_{\min }=\min _{u} \beta_{u}\left(\beta_{\min }>1\right)$ определяет область $\tilde{X}$, точки которой удовлетворяют условию

$$
\frac{N}{\beta_{\min }-1} \sum_{i=1}^{p} \sum_{h=1}^{p}\left(x_{i}-\bar{x}_{i}\right)\left(x_{h}-\bar{x}_{h}\right) a^{i h} \leqslant 1 .
$$

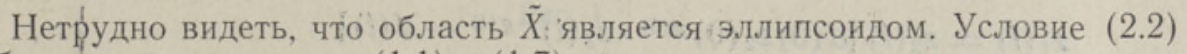
добавляется к условиям $(1.1)-(1.7)$ задачи управления.

\section{3. Модель $\dot{M}_{t}$}

При конструировании теоретической модели используются теоретические основы каталитических процессов в комбинации со статистическим анализом промышленного объекта.

Сложность явлений, происходящих в слое катализатора, исключает возможность постадийного изучения диффузии, адсорбции и кинетики реакций, в связи с чем приходится ограничиться формальным описанием макрокинетики реакций, наличие которых подтверждается анализом контактного газа.

Предполагаются гомогенный слой идеального вытеснения и установившийся поток газов.

Примем следующую схему реакций, отличающуюся от принятой ранее [3] только предположением, что СО получается при термическом распаде формальдегида:

1. $\mathrm{CH}_{3} \mathrm{OH}+{ }^{1} /{ }_{2} \mathrm{O}_{2} \stackrel{k_{1}}{\longrightarrow} \mathrm{CH}_{2} \mathrm{O}+\mathrm{H}_{2} \mathrm{O}$

2. $\mathrm{CH}_{3} \mathrm{OH} \stackrel{k_{2}}{\longrightarrow} \mathrm{CH}_{2} \mathrm{O}+\mathrm{H}_{2}$

3. $\mathrm{CH}_{2} \mathrm{O} \stackrel{k_{3}}{\longrightarrow} \mathrm{CO}+\mathrm{H}_{2}$

4. $\mathrm{CH}_{3} \mathrm{OH}+3 /{ }_{2} \mathrm{O}_{2} \stackrel{k_{4}}{\longrightarrow} \mathrm{CO}_{2}+2 \mathrm{H}_{2} \mathrm{O}$ 
Кажущиеся коэффициенты скоростей $\kappa_{1}, \kappa_{2}, \kappa_{3}, \kappa_{4}$ являются не только чисто кинетическими коэффициентами, но учитывают также лимитирующий эффект диффузии. Стационарное распределение концентраций по высоте слоя катализатора $l(0 \leqslant l \leqslant 1)$ при линейной скорости газов $v$ можно представить уравнениями материального баланса

$$
\begin{aligned}
v \frac{d z_{1}}{d l} & =k_{2} z_{2}+k_{1} z_{2} z_{3}-k_{3} z_{1} \\
-v \frac{d z_{2}}{d l} & =k_{2} z_{2}+\left(k_{1}+k_{4}\right) z_{2} z_{3} \\
-2 v \frac{d z_{3}}{d l} & =\left(k_{1}+k_{4}\right) z_{2} \\
v \frac{d z_{4}}{d l} & =k_{4} z_{2} z_{3},
\end{aligned}
$$

в которых $z_{1}, z_{2}, z_{3}^{2}, z_{4}$ - концентрации $\mathrm{CH}_{2} \mathrm{O}, \mathrm{CH}_{3} \mathrm{OH}, \mathrm{O}_{2}$ и $\mathrm{CO}_{2}$ соответственно.

Учитывая факт прохождения реакций во внешнедиффузнонной областн, принимается порядок реакций не выше 1,5 .

Аналитическое интегрирование системы (3.2) дает следующие соотношения:

$$
\begin{aligned}
z_{1}(l) & =z_{2}(l)\left[d(0) z_{2}(0) g(0) \cdot z_{2}(l)-g(l)-d(l)\right] \\
z_{2}(l) & =z_{2}(0) \frac{(1-R)^{2} e^{-C l}}{\left(1-R e^{-C l}\right)^{2}}, \quad z_{3}(l)=-a+B \frac{1+R e^{-C l}}{\left(1-R e^{-C l}\right)^{2}} \\
z_{4}(l) & =\frac{k_{4} z_{2}(0)}{v C R}\left\{\frac{(1-R)^{2}\left[\left(1-R e^{-C l}\right)(B+a)-B\right]}{\left(1-R e^{-C l}\right)^{2}}-\right. \\
& -(1-R)(B+a)+B\} \\
a & =\frac{k_{2}}{k_{1}+k_{4}}, \quad R=\frac{A-B}{A+B} \\
A & =a+z_{3}(0), \quad B=\sqrt{A^{2}-z_{2}(0)}, \quad C=\frac{k_{1}+k_{4}}{v} B \\
d(l)= & \frac{k_{2}+k_{1} z_{3}(l)}{k_{2}-k_{3}+\left(k_{1}+k_{4}\right) z_{3}(l)}, \quad d(0)=d(l) \mid l=0 \\
g(l)= & \frac{k_{2}-k_{3}+\left(k_{1}+k_{4}\right) z_{3}(l)}{k_{2}+\left(k_{1}+k_{4}\right) z_{3}(l)}, \quad g(0)=g(l) \mid l=0 .
\end{aligned}
$$

Система (3.3) при $\quad z_{1 \mid l=0}=0,\left.\quad z_{2}\right|_{l=0}=z_{2}(0),\left.\quad z_{3}\right|_{l=0}=z_{3}(0)$, $\left.z_{4}\right|_{l=0}=0$ определяет следующие концентрации контактного газа: $\left.z_{1}\right|_{l=1}=z_{1}(1),\left.\quad z_{2}\right|_{l=1}=z_{2}(1),\left.\quad z_{3}\right|_{l=1}=z_{3}(1), \quad z_{4 \mid l=1}=z_{4}(1)$. 
Зная фактические концентрации $z_{2}(0), z_{3}(0), z_{1}(1), z_{2}(1), z_{3}(1)$, $z_{4}(1)$ и скорость $v$, можно вычислить кажущиеся коэффициенты скоростей реакций $k_{1}, k_{2}, k_{3}, k_{4}$, удовлетворяющие системе (3.3). В таблице (см. приложение) приводятся значения коэффициентов скоростей, вычисленные для каждой смены одного цикла работы катализатора. Графики функций $z_{1}(l), z_{2}(l), z_{3}(l)$ (3.3) при средних для того же цикла значениях $k_{1}, k_{2}, k_{3}, k_{4}, v, z_{2}(0), z_{3}(0)$ и при $k_{3}=0,5,10$ изображены на рис. 1.

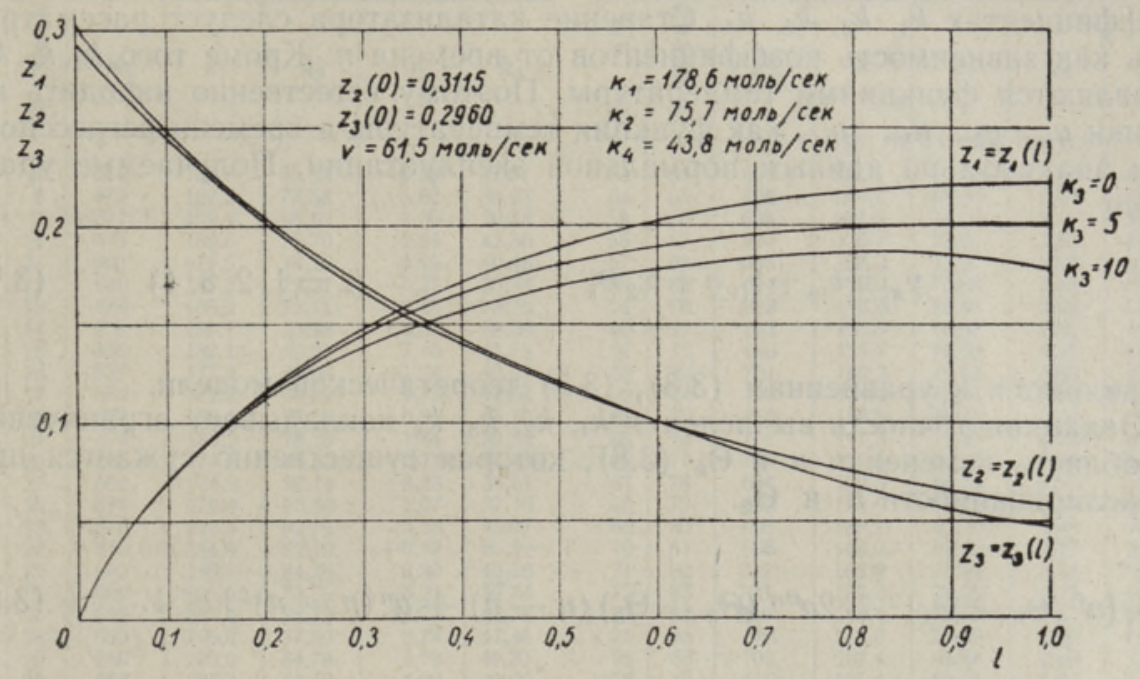

Рис. 1.

Уравнение теплового баланса учитывает приход физической теплоты $Q_{s}=c_{p s} v \Theta_{s}$, унос теплоты с контактным газом $Q_{v}=c_{p v} v \Theta_{v}$ при соответствующих теплоемкостях $c_{p}$, температурах $\Theta$ и скорости газа $v$ и выделяемое в реакциях количество теплоты $Q_{r}$.

Приближенно

$$
Q_{r}=H_{1} k_{1} \bar{z}_{2} \bar{z}_{3}+H_{2} k_{2} \bar{z}_{2}+H_{3} k_{3} \bar{z}_{1}+H_{4} k_{4} \bar{z}_{2} \bar{z}_{3}
$$

где $H_{i}$ теплоты отдельных реакций; $\bar{z}_{i}=\int_{0}^{1} z_{i}(l) d l-$ средние значения концентраций по высоте слоя.

Пренебрегая тепловыми потерями в реакторе, можно выписать уравнение теплового баланса

$$
Q_{s}+Q_{r}+Q_{v}=0
$$

Фактическая температура контактного газа $\Theta_{v}$ на выходе из слоя катализатора не измеряется. Поэтому необходимо $\Theta_{v}$ вычислять косвенно, путем введения температурного коэффициента $\xi=\frac{\Theta_{v}}{\Theta_{k}}$. Интегрирование функций $z_{i}(l)(3.3)$ и подстановка результата в (3.4) и (3.5) дает после несложных преобразований выражение для оценки измеряемой температуры $y_{\Theta_{k}}$ в функции начальных концентраций 


$$
\begin{gathered}
y_{\Theta_{k}}=\frac{1}{c_{p v} v \xi}\left\{c_{p s} v \Theta_{s}+\left\{\left(H_{1} k_{1}+H_{4} k_{4}\right)\left[-a+B\left(1+\frac{2}{C} \ln \frac{1-R e^{-C}}{1-R}\right)\right]-\right.\right. \\
\left.\left.-H_{2} k_{2}\right\} \times \frac{z_{2}(0)(1-R)}{R C}\left(1-\frac{1-R}{1-R e-C}\right)\right\}
\end{gathered}
$$

Учитывая, что $H_{3} \ll H_{1}, H_{2}, H_{4}$ и $k_{3} \ll k_{1}, k_{2}, k_{4}\left[{ }^{4}\right]$ (см. приложение), пренебрегаем теплотой реакции 3.

В принятой структуре модели свойства катализатора скрываются в коэффициентах $k_{1}, k_{2}, k_{3}, k_{4}$. Старение катализатора следует рассматривать как зависимость коэффициентов от времени $n$. Кроме того, $k_{1}, k_{2}, k_{3}$. $k_{4}$ являются функциями температуры. Поэтому естественно находить их оценки $y_{k_{1}}, y_{k_{2}}, y_{k_{3}}, y_{k_{\star}}$ как функции температуры и времени регрессионным анализом по данным нормальной эксплуатации. Получаемые уравнения

$$
y_{k_{i}}=b_{i 0}+b_{i 1} n+b_{i 2} \theta_{k} \quad(i=1,2,3,4)
$$

добавляются к уравнениям (3.3), (3.5) теоретической модели.

Заданная точность вычисления $k_{1}, k_{2}, k_{3}, k_{4}$ накладывает ограничения на область изменения $n$ и $\Theta_{k}(3.8)$, которая существенно сужается при коррелированности $n$ и $\Theta_{k}$.

$$
\frac{N}{\beta-1}\left[a^{\Theta}\left(\Theta_{k}-\bar{\Theta}_{k}\right)^{2}+2 a^{\Theta n}\left(\Theta_{k}-\bar{\Theta}_{k}\right)(n-\bar{n})+a^{n}(n-\bar{n})^{2}\right] \leqslant 1 .
$$

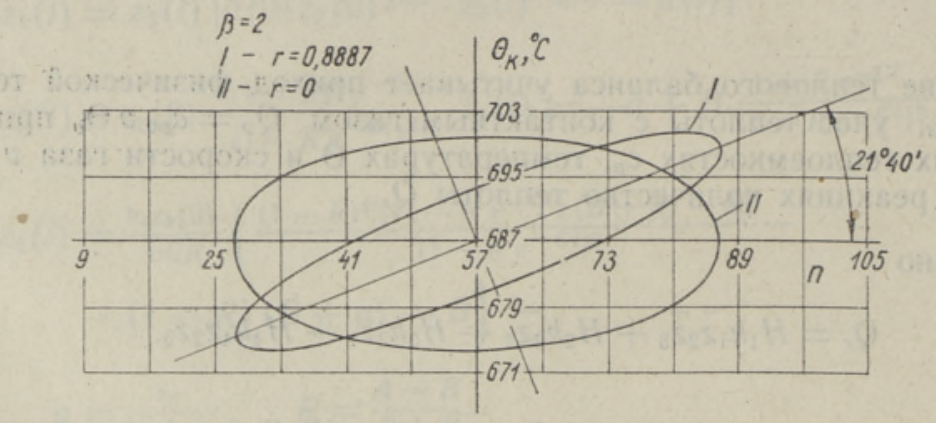

Рис. 2.

График границы области (3.8) для одного из циклов работы катализатора приведен на рис. 2. Условие (3.8) добавляется к (1.1) - (1.7) при решении задачи управления на основе модели $M_{t}$.

Справедливы следующие соотношения:

$$
\eta_{\Phi}=\frac{z_{1}(1)}{z_{2}(0)}, \quad \eta_{g}=\lambda \eta_{\Phi} v z_{2}(0), \quad \eta_{\gamma}=\frac{z_{2}(1)}{z_{1}(1)}
$$

позволяющие использовать $M_{t}$ в решении задачи $(1.1)-(1.7)$. 
ПРИЛОЖЕНИЕ

Модель $M_{t}$

Значения коэффициентов скоростей, вычисленные для каждой смены одного цикла катализатора

\begin{tabular}{|c|c|c|c|c|c|c|c|c|c|c|c|c|c|}
\hline$\frac{E}{E}$ & $\begin{array}{l}\frac{3}{3} \\
\frac{1}{2} \\
\dot{2}\end{array}$ & $\begin{array}{l}\left.t: A_{i}\right) \\
s: ;\end{array}$ & $k_{1}$ & $k_{2}$ & $k_{3}$ & $k_{4}$ & $\underline{E}$ & $\begin{array}{l}\frac{3}{3} \\
\frac{3}{2} \\
\text { 일 }\end{array}$ & $\Theta_{k}$ & $k_{1}$ & $k_{2}$ & $k_{3}$ & $k_{1}$ \\
\hline 1 & 7 & 661 & 139.7 & 65,83 & $-0,18$ & 32,01 & 53 & 64 & 698 & 171.5 & 69.55 ? & 3,62 & 44.89 \\
\hline 2 & 8 & 675 & 162,3 & 77,58 & 5,60 & 38,43 & 54 & 65 & 700 & $\begin{array}{l}171,0 \\
171,2\end{array}$ & 68,55 & $\begin{array}{l}3,02 \\
0,50\end{array}$ & $\begin{array}{l}44,09 \\
41,96\end{array}$ \\
\hline 3 & . 9 & 6. $671^{1+4}$ & 159.4 & 65.62 & 1,39 & 30,48 & 55 & +6 & 694 & 169.5 & 74,00 & 2,45 & 39,93 \\
\hline 4 & 10 & 653 & 189.5 & 76,76 & 2,51 & 42,50 & 56 & 67 & 697 & 170.7 & 73,50 & 3,53 & 48,68 \\
\hline 5 & 11 & 660 & 179.5 & 74,26 & 0,16 & 40,48 & 57 & 68 & GSS & 160,3 & 72,97 & 2,72 & 46,78 \\
\hline 6 & 12 & 660 & 165.2 & 72,22 & $-1,27$ & 35.74 & 58 & 69 & 694 & 170,9 & 73,68 & 3,80 & 42,28 \\
\hline 7 & 13 & 660 & 185,2 & 72,71 & -0.43 & 38,39 & 59 & 70 & 688 & 156,6 & 75,54 & 2,63 & 47.24 \\
\hline 8 & 14 & 6.59 & 186,1 & 73,48 & 0,51 & 38,58 & 60 & 71 & 692 & 185,9 & 76,00 & 5.60 & 42.61 \\
\hline 9 & 15 & 658 & 189,1 & 89,57 & 7,40 & 41,78 & 61 & 72 & 696 & 158,7 & 74,50 & 4,34 & 43,21 \\
\hline 10 & 16 & 658 & 177,4 & 73.00 & $-1,24$ & 40,77 & 62 & 73 & 701 & 162,3 & 72,28 & 3,80 & 45,08 \\
\hline 11 & 17 & 662 & 181,3 & 73.39 & 1,73 & 41,54 & 63 & 74 & 700 & 174,6 & 75,91 & 4,23 & 43,38 \\
\hline 12 & 18 & 670 & 188,1 & 77.95 & 0,83 & 36.30 & 64 & 75 & 699 & 153,5 & 68,18 & 2,00 & 42,83 \\
\hline 13 & 19 & 674 & 183,2 & 78,20 & 1,64 & 39,55 & 65 & 76 & 701 & 141,3 & 71,41 & 0,14 & 45,17 \\
\hline 14 & 20 & 663 & 174,6 & 75.82 & 0,74 & 37,41 & 66 & 77 & 700 & 151,3 & 63,90 & 2,90 & 39,42 \\
\hline 15 & 21 & 662 & 174,9 & 90,14 & 6,23 & 39,51 & 67 & 78 & 696 & 159.5 & 69,09 & 5,06 & 39.51 \\
\hline 16 & 22 & 674 & 176,0 & 73,89 & 1,07 & 37,10 & 68 & 79 & 693 & 138,6 & 65,72 & 2,54 & 43.95 \\
\hline i7 & 23 & 673 & 210,0 & 92,76 & 5,33 & 43,07 & 69 & 80 & 696 & 156,2 & 63.79 & 3,44 & 41,07 \\
\hline 18 & 24 & 678 & 184,4 & 72,16 & -0.97 & $35.59^{\circ}$ & 70 & 81 & 695 & 148,2 & 63,60 & 2,27 & 36,48 \\
\hline 19 & 25 & 680 & 189,9 & 84.28 & 8,30 & 40,68 & 71 & 82 & 702 & 165,9 & 71,88 & 2,63 & 50,24 \\
\hline 20 & 26 & 680 & 191,4 & 73,75 & -3.22 & 39.74 & 72 & 83 & 700 & 185,3 & 74,92 & 4,25 & 48,14 \\
\hline 21 & 27 & 682 & 207.7 & 78,72 & $-2,77$ & 43,78 & 73 & 84 & 698 & 186,4 & 76,07 & 1,41 & 48,41 \\
\hline 22 & 28 & 680 & 195,6 & 77.59 & 0,74 & 41,46 & 74 & 85 & 698 & 179,0 & 76,39 & 3.80 & 49,35 \\
\hline 23 & 29 & 680 & 200,6 & 84,78 & 4,70 & 40,30 & 75 & 86 & 701 & 207,4 & 91,88 & 7,40 & 57,18 \\
\hline 24 & 30 & 683 & 227,1 & 84,40 & 3,26 & 49,66 & 76 & 57 & 699 & 195,4 & 80,35 & 2,07 & 52,17 \\
\hline 25 & 31 & 680 & 206,6 & 83,15 & 2,63 & 44,91 & 77 & 88 & 693 & 182,0 & 89,81 & 9,20 & 47,60 \\
\hline 26 & 32 & " 680 & 206.1 & 86.82 & 3,17 & 43,08 & 78 & 89 & 700 & 184,4 & 84,55 & 5,60 & 47,19 \\
\hline 27 & 33 & 681 & 197,8 & 83.32 & 3,26 & 43,27 & 79 & 90 & 690 & 198,3 & 79,71 & 2,00 & 51,54 \\
\hline 28 & 34 & 679 & 182,8 & 75.77 & 2,40 & 41,59 & 80 & 91 & 690 & 195,4 & 80,26 & 3,80 & 48,32 \\
\hline 29 & 36 & 686 & 201,9 & 80.45 & 0,70 & 46,19 & 81 & 92 & 690 & 203.5 & 88,11 & 6,05 & 54,92 \\
\hline 30 & 38 & 680 & 200,0 & 80,81 & $-0,70$ & 42,40 & 82 & 93 & 694 & 187,3 & 84,68 & 4,70 & 57,26 \\
\hline 31 & 39 & 681 & 207.7 & 85.49 & 4,16 & 47,87 & 83 & 94 & 697 & 182,7 & 85,74 & 7,40 & 49,92 \\
\hline 32 & 40 & 684 & 162,4 & 76,34 & 5,60 & 44,84 & 84 & 95 & 697 & 174,7 & 75,84 & 3,80 & 51,95 \\
\hline 33 & 41 & 679 & 125,8 & 59,80 & 2,90 & 33,06 & 85 & 96 & 697 & 182,0 & 83,38 & 6,14 & 53,38 \\
\hline 34 & 42 & 696 & 175,9 & 68,62 & 0,83 & 40,36 & 86 & 99 & 699 & 197.7 & 80.64 & 3.80 & 55,89 \\
\hline 35 & 43 & 681 & 192,3 & 69,33 & $-1,06$ & 42,86 & 87 & 100 & 696 & 190,8 & 76,13 & 2.54 & 56,40 \\
\hline 36 & 44 & 679 & 185,2 & 77,25 & 3,21 & 43,19 & 88 & 101 & 697 & 190.9 & 77,47 & 5.60 & 53,95 \\
\hline 37 & 45 & 698 & 192,7 & 80.09 & 2,54 & 45,34 & 89 & 102 & 693 & & 41 & 1,44 & 49,96 \\
\hline 38 & 46 & 684 & 189.7 & 77,73 & 0.27 & 43,95 & 90 & 103 & 707 & 159,1 & 67.09 & 2,05 & 47.80 \\
\hline 39 & 47 & 685 & 190.6 & 82,36 & 3,53 & 45,19 & 91 & 104 & 706 & 167,3 & 70,71 & 3,80 & 47,06 \\
\hline 40 & 48 & 683 & 189,6 & 81,14 & 3,50 & 43,74 & 92 & 105 & 702 & 150,8 & 83,65 & 6,50 & 58,17 \\
\hline 41 & 49 & 683 & 190, & 82,6 & 3,80 & 45 & 93 & 106 & 702 & & & & 50.00 \\
\hline 42 & 50 & 683 & 188,6 & 79,39 & 1,82 & 46,64 & 94 & 107 & 704 & & 81,45 & 5,60 & 50,30 \\
\hline 43 & 52 & 691 & 205,8 & 80,82 & 2,72 & 46,66 & 95 & 109 & 704 & 144,4 & 63,70 & 7,00 & 46,79 \\
\hline 44 & 53 & 687 & 211,1 & 85,31 & 5,60 & 48,39 & & & & & & & \\
\hline 45 & 54 & 685 & 194,2 & 78,33 & 3,08 & 47,48 & & & & & & & \\
\hline 46 & 56 & 684 & 182,9 & 80.3 & 5.60 & 45,7 & & & & & & & \\
\hline 47 & 57 & 687 & 183,4 & 78,63 & 3,51 & 49,80 & & & & & & & \\
\hline 48 & 58 & 692 & 182,1 & 73,76 & 3,17 & 44,31 & & & & & & & \\
\hline 49 & 59 & 700 & 109,6 & 76,46 & 3,62 & 34.75 & & & & & & & \\
\hline 50 & 60 & 694 & 175,1 & 81,68 & 4,34 & 42,19 & & & & & & & \\
\hline $\begin{array}{l}51 \\
52\end{array}$ & $\begin{array}{l}62 \\
63\end{array}$ & $\begin{array}{l}689 \\
694\end{array}$ & $\begin{array}{l}163,3 \\
161,9\end{array}$ & $\begin{array}{l}74,33 \\
64,91\end{array}$ & $\begin{array}{l}4,43 \\
0,10\end{array}$ & $\begin{array}{l}40,15 \\
39,05\end{array}$ & & & & & & & \\
\hline & & 057 & & & & & & & & & & & \\
\hline
\end{tabular}

Коэффициенты корреляции

\begin{tabular}{c|c|c|c|c}
\hline & $k_{1}$ & $k_{2}$ & $k_{3}$ & $k_{4}$ \\
\hline$\stackrel{\Theta}{k}^{n}$ & $-0,1929$ & $-0,1135$ & - & 0,7181 \\
& $-0,2193$ & $-0,0965$ & 0,3786 & 0,5654
\end{tabular}


$k_{1}$ и $k_{2}$ считаем константными

$$
k_{1}=178,6 \pm t_{\frac{\alpha}{2}, N-1} \frac{19,77}{\sqrt{95}}, \quad k_{2}=75,7 \pm t_{\frac{\alpha}{2}, N-1} \frac{6,05}{\sqrt{95}} .
$$

Уравнения регрессин

$$
y_{k_{3}}=-45,69+0,07098 \boldsymbol{\theta}_{k^{\prime}}, \quad y_{k_{\mathrm{t}}}=137,7+0,1951 n-0,1521 \quad \boldsymbol{\theta}_{k_{k}} .
$$

Матрица коэффициентов ковариации и обратная к ней матрица

$$
\begin{aligned}
& A=10^{4}\left\|\begin{array}{ll}
8,432 & 3,243 \\
3,243 & 1,578
\end{array}\right\| \quad A^{-1}=10^{-4}\left\|\begin{array}{rr}
0,5659 & -1,162 \\
-1,162 & 3,022
\end{array}\right\| \\
& \Theta_{s}=120^{\circ} \mathrm{C}, \quad c_{p s}=43,2, \quad c_{p 0}=64,8 \text { дж/моль град } \\
& H_{1}=156,7 \cdot 10^{3}, \quad H_{2}=-85,33 \cdot 10^{3}, \quad H_{3}=6,113 \cdot 10^{3}, \quad H_{4}=676,6 \cdot 10^{3} \text { дж/моль град } \\
& \xi=0,2212 \pm t_{\frac{\alpha}{2}, N-1} \frac{0,08185}{\overline{\sqrt{95}}}, \quad \lambda=2,335 .
\end{aligned}
$$

\section{Л И ТЕ Р А Т Р А}

1. Понтрягин Л. С., Болтянский В. Г., Гамкрелидзе Р. В., Мищен к о Е., Математическая теорня. оптимальных процессов, М., 1961.

2. Willi a m s E. J., Regression analysis, New York, John Wiley, 1959.

3. Y a m a u ch i S., J. Soc. Org. Synth. Chem. Japan, 18, No. 6, 592-601 (1960).

4. Handbook of Chemistry and Physics, 37-th ed., Ed. in chief Ch. D. Hodgman, Chemical Rubber Publishing Co., 1955.

Институт кибернетики

Академии наук Эстонской ССР

\section{Поступила в редакцию} 16/IV 1965

\section{R. TAVAST, U. JAAKSOO}

\section{FORMALDEHUUDI TOOTMISPROTSESSI OPTIMAALNE JUHTIMINE. I}

Formuleeritakse kvaasistatsionaarse katalüütilise protsessi juhtimisülesanne. Koostatakse protsessi empiiriline ja teoreetiline mudel ning esitatakse nende täpsuse hindamise. metoodika.

\section{R. TAVAST, U. JAAKSOO}

\section{THE OPTIMAL CONTROL OF PRODUCTION OF FORMALDEHYDE FROM METHANOL. I}

The first part of the paper deals with formulation of the optimal control problem of a quasistationary catalytic process. Empiric and theoretic models of the process are constructed and the methods for estimating of their adequate accuracy are given. 